\title{
Comparing Typical Opening Move Choices Made by Humans and Chess Engines
}

\author{
Mark Levene \\ School of Computer Science and Information Systems \\ Birkbeck College, University of London \\ London WC1E 7HX, U.K. \\ mark@dcs.bbk.ac.uk \\ Judit Bar-Ilan \\ Department of Information Science \\ Bar-Ilan University, Israel \\ barilaj@mail.biu.ac.il
}

\begin{abstract}
The opening book is an important component of a chess engine, and thus computer chess programmers have been developing automated methods to improve the quality of their books. For chess, which has a very rich opening theory, large databases of highquality games can be used as the basis of an opening book, from which statistics relating to move choices from given positions can be collected. In order to find out whether the opening books used by modern chess engines in machine versus machine competitions are "comparable" to those used by chess players in human versus human competitions, we carried out analysis on 26 test positions using statistics from two opening books one compiled from humans' games and the other from machines' games. Our analysis using several nonparametric measures, shows that, overall, there is a strong association between humans' and machines' choices of opening moves when using a book to guide their choices.
\end{abstract}

\section{Introduction}

Computer chess has been an active area of research since Shannon's Sha50 seminal paper, where he suggested the basic minimax search strategies and heuristics, which have been refined and improved over the years. The many advances since then in improving the search engine algorithms, the static evaluation of chess positions, the representation and learning of chess knowledge, the use of large opening and endgame databases, and the exploitation of computer hardware including parallel processing and special-purpose hardware, have resulted in the development of powerful computer-chess programs, some of which are of top-Grandmasterlevel strength New02.

The ultimate test for a chess-playing program is to play a match against the World Champion or one of the leading Grandmasters. In May 1997 an historic match was played between between IBM's Deep Blue chess computer and Garry Kasparov, then World Champion, resulting in a spectacular win for the machine, 3.5 - 2.5. (See Www.chess.ibm.com for the archived web site of this historic match.) Despite the computer winning the match there has 
been an ongoing debate since then on whether the highest ranking computer-chess programs are at the world-championship level, through most seem to agree that it is inevitable that eventually chess machines will dominate. Using special-purpose hardware and parallelization Deep Blue New02 was capable of analysing up to 200 million positions per second, while the only other chess program to-date, known to run at a comparable speed, is Hydra DL05a. The Hydra team, consider their program to be successor of Deep Blue and their goal is to create the strongest chess-playing computer, which can convincingly defeat the human world chess champion. A recent six game match played in June 2005 between Hydra and leading British Grandmaster Michael Adams resulted in a convincing win for the machine, 5.5 - 0.5. (See http://tournament.hydrachess.com for the web site archiving the match.)

There have been other recent man-machine chess matches against top performing multiprocessor chess engines capable of analysing several million positions per second, with the results against world champions still being inconclusive Pra03]. It remains to be seen whether the era of chess man-machine contests is nearing its end, nonetheless, with machines having ever growing computing resources the future looks bleak for any human contestants in such matches.

Here we concentrate on the late opening/early middle-game phase of the game, and, in particular the research question we address is whether the opening books used by modern chess engines in machine versus machine competitions are "comparable" to those used by chess players in human versus human competitions.

For humans, opening preparation is known to be very important, as can be seen, for example, by the large proportion of chess books concentrating on the opening phase of the game. Modern chess players also use software packages, such as those developed by ChessBase (Www.chessbase.com) or Chess Assistant (www.chessassistant.com), to assist them in their opening preparation for matches. These packages typically make use of large databases of opening positions, referred to as opening books, whose positions can be searched and are linked to recent databases of games (some of which may be annotated by experts). This combined with the use of state-of-the-art chess engines for position analysis, provides players with extremely powerful tools for opening study and preparation. It is often recommended that chess students combine the study of openings with typical middle game motifs and endgame structures which may arise from the openings in question, and computer chess software can be very useful for this purpose JAE03.

Opening theory has become so developed that it is common between expert chess players to play the first 15 moves or so from memory; see, for example the Encyclopedia of Chess Openings marketed by Chess Informant (www.sahovski.co.yu). As an antidote to the study of opening theory the former world chess champion, Bobby Fischer, suggested a chess variant known as Fischer Random chess or Chess960 (www.chessvariants.org/diffsetup.dir/ fischer.html), where the initial position of the chess pieces is randomised. Due to the 960 different starting positions in Chess960, knowledge of current chess opening theory is not very useful, and thus the strongest player will win without having to memorise lengthy opening variations. Chess960 is becoming a popular variant of chess but, at least in the near future, it is unlikely to replace classical chess which still fascinates millions world wide.

As the top chess engines now compete at Grandmaster level, the opening book has become an important feature contributing to their success. These days it quite normal for an opening book specialist to be an integral part of the development team of a chess engine. As an 
example of computer chess opening preparation, back in 1995 Fritz 3 defeated a prototype of Deep Blue in the World Computer Chess Championship when Deep Blue made a crucial mistake as it went out of its opening book and had to assess the position using its search and evaluation engine. For its matches against Garry Kasparov in 1997, the Deep Blue team included Grandmaster Joel Benjamin, who was responsible for developing Deep Blue's knowledge and fine tuning its opening book [New02]. Since then it has become common practice to include a Grandmaster level chess player in the chess engine's team especially for high profile man-machine matches.

Due to the importance of the opening book as a component of a chess engine, computer chess programmers have been developing automated methods for improving the quality of their books. For a game such as chess, having a rich and well developed opening theory, a good starting point for building an opening book is to have available a large database of high-quality games with a sizeable proportion of recent game records. Game statistics including a summary of the game results when the move was played, the popularity of the move in the database, how strong are the players of the move, and how recently the move was played, can be weighted to produce an evaluation of the "goodness" of a move that can inform the chess engine's evaluation function. Deep Blue utilised these statistics to automatically extend its relatively small hand-crafted opening book, which consisted of about 4000 positions CHH02, and, similarly Hydra extends its relatively small opening book, typically containing about 10 moves per variation (www.hydrachess.com/hydrachess/faq.php). While Deep Blue combines the "goodness" factors in a non-linear fashion in order to influence the choice of move in the absence of information from its opening book Cam99, Hydra combines the "goodness" factors in a linear fashion to influence the choice of playable moves [DL05b]. An opening book and its extension constructed by the above method will not be perfect, simply due to the fact that opening theory is still dynamic, and the statistics often reflect what is fashionable rather than what is objectively best. Hydra takes this factor into account by adjusting the thinking time of a move when the engine chooses a book move in preference to a move selected by the engine.

In games such as Awari, where large databases of games do not exist, the above techniques cannot be applied, so the opening book needs to be constructed by using the game engine to perform deep searches and generate the evaluation of the positions to be stored in the book [Lin00]. A best-first search method was proposed by Buro [Bur99], where at each level the move with the highest score is expanded first. In this way bad moves are ignored and no human intervention is necessary. However, this method ignores moves that are not much worst than the best move, thus allowing an opponent to "drop out" of the book after a few moves and forcing the chess engine to assess positions using its search and evaluation algorithms. To avoid this situation Lincke Lin00 proposed to expand moves in such a way that priority is given to moves at lower search levels, whose score is within a tolerance level from the best move.

It is also useful to incorporate some form of learning to tune the opening book in order to avoid playing the same mistake repeatedly. A method developed by Hyatt Hya99 looks at the next ten move evaluations in games it played after the opening book was left, and extrapolates an approximation of the true value to store in the book as a learnt value for the position. The learning is conditioned upon the depth of searches that produced the learnt value, the strength of the opposition in the game played, and whether the engine or its opponent made a mistake. 
We now give an overview of the experiment we have carried out. For the purpose of data analysis we used the Nunn2 test suite devised by Grandmaster Dr. John Nunn to test chess engines' strength on a variety of late opening/early middle-game positions; the Nunn2 test is distributed by ChessBase together with its chess engine, Fritz. The Nunn2 test was chosen, since its 25 positions arise from a variety of openings with different characteristics, and for all these positions there are several reasonable candidate moves. We augmented the Nunn2 test with the initial position, as we were interested to find out which first moves do humans and machines prefer.

To compare the choices of humans to those of engines, we made use of two high-quality opening books: Powerbook 2005, marketed by ChessBase, derived from a large collection of human versus human games, and Comp2005 derived from a large collection of machine versus machine games compiled by Walter Eigenmann (www.beepworld.de/members38/eigenmann). For each position we collected the statistics related to the move choices for the position from both opening books, including the rank of each move choice, the number of games in the database in which the move choice was played, and the percentage score achieved for this choice.

The analysis we carried out from the data compared both the distribution of the move choices and the ranks of the choices implied by this distribution. The ranks of the moves made by humans and engines were compared using a nonparametric association measure we have used in previous studies, where we compared move choices of different chess engines [LB05] and the ranking of search results by web search engines BML06. The measure is a weighted version of Spearman's footrule [FKS03], which we call the M-measure. The distributions of move choices were compared using the Jensen-Shannon divergence (JSD) nonparametric measure $\mathrm{GBC}^{+} 02$, ES03, which allows us to measure the similarity between two distributions. In addition, for each position we computed the degree of overlap between move choices of humans and engines and the expected percentage score for the position, i.e. out of the total number of games played from the position what was the percentage of wins and draws.

The results show a surprisingly close association between humans' and machines' opening books. The M-measure is over 0.75 while the JSD is just above 0.70 , on average, on a scale between 0 and 1. It is also shown that, apart from two outliers, the M-measure and JSD are highly correlated with a correlation coefficient just above 0.65 . Moreover, the degree of overlap between move choices is also just above 0.60 on average, so despite the strong association between humans' and machines' choice of opening moves there are also differences, although these disparate moves do not tend to be the highly ranked moves. Finally, for the positions we investigated, the expected scores from white's point of view were similar, on average over $55 \%$, for both humans and machines, which indicates a significant advantage to white.

The rest of the paper is organised as follows. In Section 2 we describe the measures we used to compare the rankings induced by the two opening books. In Section of we give the detail of the data collection phase. In Section 1 we present the data analysis carried out and interpret the results. In Section 5 we discuss possible extensions and applications of the comparison techniques we have used, and finally, in Section 6 we give our concluding remarks. 


\section{The Measures}

We used several nonparametric measures GC03 to test the correspondence between the two opening books. To illustrate the measures consider the initial chess position and assume that only the top-10 move choices were recorded in Powerbook 2005 (pb) and in Comp2005 (comp). (In the experiment 20 moves were actually recorded in each opening book for the initial position.) The data collected is shown in Table 1, where the second and fourth column indicate the rank of the move in pb and comp, respectively, while the third and fifth columns indicate the popularity (i.e. the number of games in the database in which the move was played) in pb and comp, respectively; a zero entry in a column implies that no games were recorded for that move in the corresponding opening book.

\begin{tabular}{|c|c|r|c|r|}
\hline Move & R-pb & P-pb & R-comp & P-comp \\
\hline \hline e4 & 1 & 448923 & 1 & 122882 \\
d4 & 2 & 361246 & 2 & 105119 \\
Nf3 & 3 & 103542 & 3 & 20820 \\
c4 & 4 & 78408 & 4 & 20023 \\
g3 & 5 & 10142 & 5 & 3359 \\
b3 & 6 & 3252 & 7 & 1121 \\
f4 & 7 & 2754 & 8 & 945 \\
Nc3 & 8 & 1382 & 6 & 1445 \\
b4 & 9 & 718 & 0 & 0 \\
d3 & 10 & 390 & 10 & 333 \\
e3 & 0 & 0 & 9 & 493 \\
\hline
\end{tabular}

Table 1: Data for top-10.

The simplest measure we used is the degree of overlap between the two two ranked lists (Overlap). It can seen from Table 1 that the overlap is 9 out of 11, i.e. the degree of overlap is 0.818 .

The second measure we used is a weighted variation of Spearman's footrule [FKS03, which we call the M-measure BML06. From Table 11 we see that pb and comp agree on the ranking of the top-5 move choices but disagree thereafter. To compute the M-measure we assign each move with a rank greater than zero its reciprocal rank, and to all moves that did not have a rank, i.e the entry for their rank is zero, we assign a rank of one plus the maximum rank in the column that had the zero and then take its reciprocal, as shown in Table 2.

To compute the M-measure, we now sum the absolute difference of the the reciprocal ranks for each pair of corresponding moves as recorded in Table 2, normalise the result by dividing by the maximum value of the measure, and finally subtract the result from one to arrive at a similarity measure rather the a dissimilarity measure. More formally, the M-measure, is given by

$$
1-\frac{\sum_{i=1}^{n}\left(\left|\frac{1}{\operatorname{rank}_{1}(i)}-\frac{1}{\operatorname{rank}_{2}(i)}\right|\right)}{\operatorname{maxM}},
$$

where $n$ is the number of moves being compared, $\operatorname{rank}_{1}(i)$ is the rank in pb for the $i$ th move, $\operatorname{rank}_{2}(i)$ is the corresponding rank in comp for the move, and maxM is the maximum value of the measure to normalise it. The reader can verify that for our illustrative example, maxM $=4.0398$ and $\mathrm{M}=0.9694$. 


\begin{tabular}{|c|c|c|}
\hline Move & R-pb & R-comp \\
\hline \hline $\mathrm{e} 4$ & 1 & 1 \\
$\mathrm{~d} 4$ & $1 / 2$ & $1 / 2$ \\
$\mathrm{Nf3}$ & $1 / 3$ & $1 / 3$ \\
$\mathrm{c} 4$ & $1 / 4$ & $1 / 4$ \\
$\mathrm{~g} 3$ & $1 / 5$ & $1 / 5$ \\
$\mathrm{~b} 3$ & $1 / 6$ & $1 / 7$ \\
$\mathrm{f} 4$ & $1 / 7$ & $1 / 8$ \\
$\mathrm{Nc} 3$ & $1 / 8$ & $1 / 6$ \\
$\mathrm{~b} 4$ & $1 / 9$ & $1 / 11$ \\
$\mathrm{~d} 3$ & $1 / 10$ & $1 / 10$ \\
$\mathrm{e} 3$ & $1 / 11$ & $1 / 9$ \\
\hline
\end{tabular}

Table 2: Reciprocal ranks for the M-measure.

The third measure we used is the Jensen-Shannon Divergence (JSD) GBC ${ }^{+} 02$, ES03, which enables us to measure the similarity between two distributions. The first step in the computation of the JSD is to normalise the number of games played for each move by the total number of games played. For pb (column 3 of Table 1) the normalisation factor is 1,010,757, and for comp (column 5 of Table 1) the normalisation factor is 276,540. The normalised values, which can be viewed as probabilities, are shown in Table 3 . We denote the $\mathrm{pb}$ and comp probabilities by $p_{i}$ and $q_{i}$, respectively; the reader can verify the $p_{i}$ s and the $q_{i}$ s both add up to one.

\begin{tabular}{|c|c|c|}
\hline Move & P-pb $\left(p_{i}\right)$ & P-comp $\left(q_{i}\right)$ \\
\hline \hline e4 & 0.4441 & 0.4444 \\
d4 & 0.3574 & 0.3801 \\
Nf3 & 0.1024 & 0.0753 \\
c4 & 0.0776 & 0.0724 \\
g3 & 0.0100 & 0.0121 \\
b3 & 0.0032 & 0.0041 \\
f4 & 0.0027 & 0.0034 \\
Nc3 & 0.0014 & 0.0052 \\
b4 & 0.0007 & 0.0000 \\
d3 & 0.0004 & 0.0012 \\
e3 & 0.0000 & 0.0018 \\
\hline
\end{tabular}

Table 3: Normalisation of number of games played for the JSD.

The formal definition of the JSD, which is a symmetric version of the Kullback-Leibler divergence based on Shannon's entropy, is given by

$$
1-\sqrt{\frac{1}{2} \sum_{i=1}^{n_{1}} p_{i} \log _{2} \frac{2 p_{i}}{p_{i}+q_{i}}+\frac{1}{2} \sum_{i=1}^{n_{2}} q_{i} \log _{2} \frac{2 q_{i}}{p_{i}+q_{i}}},
$$

where $n_{1}$ is the number of moves recorded in pb, $n_{2}$ is the number of moves recorded in comp, and the factor of $1 / 2$ in the square root is in order to normalise the JSD. The reader can verify that for our illustrative example, JSD $=0.935$. 


\section{Data Collection}

The positions analysed were the ones from the Nunn2 test collection, augmented with the initial board configuration as position 26. The move choices made by humans were gathered from Powerbook 2005, which is an opening book marketed by ChessBase, derived from a large collection of high-class human versus human tournament games. On the other hand, the moves choice made by chess engines were gathered from Comp2005, which is an opening book we built, derived from a large collection of high-quality games played between about 2000 chess engines between 2000 and September 2005, with a time limit of at least 30 minutes per engine per game. The collection was compiled by Walter Eigenmann and the latest version can be downloaded from his web site, www.beepworld.de/members38/eigenmann. (See Table 1 for the detailed numerics for $\mathrm{pb}$ and comp.)

For each opening book and each move choice in a position we analysed, we recorded the rank, the number of games in the database in which the move was played, and the overall result achieved when the move was played in terms of the percentage score of wins and draws when the move was played (a win counts for one point and a draw for half a point).

\begin{tabular}{|l|r|r|}
\hline Opening book & \# Games & \# Positions \\
\hline \hline Powerbook (pb) & $1,011,611$ & $20,070,734$ \\
Comp2005 (comp) & 277,288 & $8,239,675$ \\
\hline
\end{tabular}

Table 4: Numerics for the opening books

\section{Data Analysis}

For each position we computed the M-measure, the JSD and the overlap; the results including maxM, are shown in Table 5. We note that when computing the JSD we have chosen to discard moves for which less than 10 games were played, as these were deemed to be statistically insignificant.

The results show a surprisingly close association between humans' and machines' opening books. The M-measure is over 0.75 while the JSD is just above 0.70 , on average, on a scale between 0 and 1 . Moreover, the degree of overlap between move choices is just above 0.60 , on average, so despite the strong association between humans' and machines' choice of opening moves there are also differences, although the disparate moves do not tend to be the highly ranked moves.

The M-measure and the JSD measure are highly correlated as can be seen from their scatter plot shown in Figure 1. Their correlation coefficient was computed as 0.5397, together with a 95\% bootstrap confidence interval [DH97] of [0.2625,0.7644]. Two obvious outliers that stand out in the scatter plot have arrows pointing to them. The outlier on the left, that has a low M-measure but high JSD, is for position 1 (Symmetrical English opening, Hedgehog variation). In this case, despite the ranking of move choices being different (low M-measure) their distribution is similar (high JSD). The outlier on the right is for position 24 (King's Indian Defence, Sämisch variation), for which there is only one move in each book with more than 9 games played, and thus the JSD is one. Moreover for this position there were three 
move choices in pb and only one in comp, so the M-measure is also very high by default. We measured the correlation coefficient after removing the outliers and it increased to 0.6549, together with a $95 \%$ bootstrap confidence interval of [0.3655, 0.8228].

We observe that Position 15 (Queen's Gambit Declined, Bf4 variation), which is the point at the bottom left of the scatter plot, is also anomalous in that there is a low association between $\mathrm{pb}$ and comp. The overlap percentage for this position is less that 0.5 , which is relatively low. In particular, there are 7 move choices in pb and 12 move choices in comp, 6 of which overlap. It is interesting to note that this variation is much more popular with computers than humans, as there were 1022 games recorded for this positions in comp while only 144 games were present in pb. Moreover, as can be seen in Table 6 below, humans perform significantly better in this position than machines, with an expected percentage score of $57.443 \%$ as opposed to $50.573 \%$.

Table 6 shows the expected percentage score for each position from the Powerbook and Comp2005 data sets from white's point of view, and the number of games recorded in the corresponding databases in which the position occurred. Near each position number in the first column we indicate whether it was white to move (w) or black (b); it can be seen that only in 7 out of the 26 positions was it black's turn to move. We note that, as with the JSD, in our computation of the expected score we have chosen to discard moves for which less than 10 games were played, as these were deemed to be statistically insignificant.

For the positions we investigated, the expected scores from white's point of view, were similar, on average over 55\%, for both humans and machines, which indicates a substantial advantage to white in most of the positions from the Nunn test. Despite this advantage for white, it is worth noting that the variance of the expected score is rather high; see the last row in Table 6 .

It seems that the distribution of move choices is consistent with an exponential distribution, since there are, generally, only very few popular move choices and the decrease in popularity is thereafter exponential, although for most positions the number of choices is too small to reach a definite conclusion. For position 26, for which we have 20 move choices in each book, we fitted an exponential distribution to the sample distribution induced by the popularity of the move choices in each book, resulting in fits with $r^{2}$ (the square of the correlation coefficient) values of 0.9535 and 0.9299 , respectively, for pb and comp.

\section{Discussion}

Possible extensions and applications of the comparison techniques we have presented are:

(1) Applying the technique to more comprehensive test sets such as the Don Dailey test GBM05, which consists of 200 positions all of which are 5 moves for each player from the initial position. A more principled approach could also be taken by collecting positions from the Encyclopedia of Chess Openings classification system.

(2) Comparing opening books of two individuals, be they human or machine. To carry out such a comparison we need to have game databases of sufficient size, from which we can construct the respective opening books. 


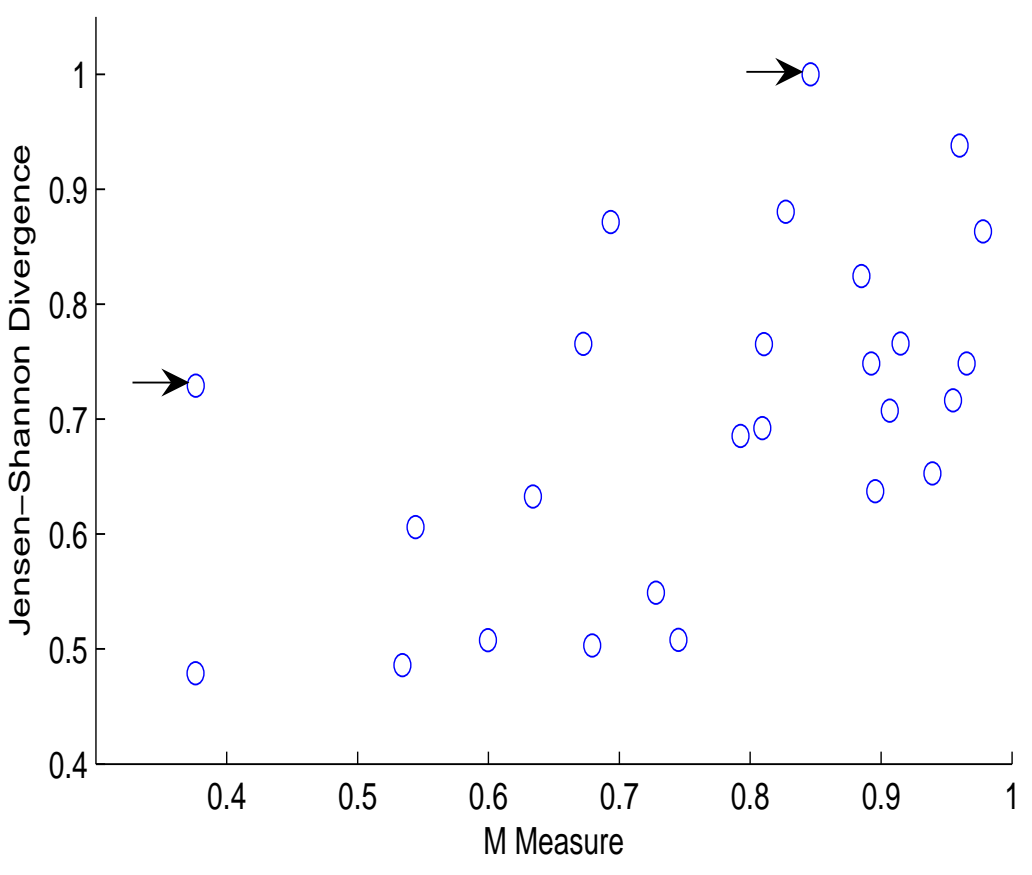

Figure 1: Scatter plot.

(3) Comparing how an opening book changes over time. For example, we could compare Powerbook 2005 to the newer Powerbook 2006.

(4) Extend the technique to middle game and endgame positions with the aid of test sets such as the WM test of Gurevich and Schumacher of positions from world champion games; the WM test can be downloaded from www.computerschach.de. This is more applicable to comparing the move choices of two available chess engines, which can display the ranking of the top-n move choices being considered, since, in general, there may be several reasonable moves from such positions and in game records we have access only to the move that was chosen.

(5) Applying the similarity M-measure to tuning the weights of evaluation function features such as material balance, mobility, development, pawn structure, and king safety [Für01] to those of a specific chess engine. The principle underlying such a technique is to compare, via the M-measure, the top-n move choices of the evaluation function we are training to the top-n move choices of the chess engine we are learning from, and to apply a gradient descent (or hill climbing) method to adjust the weights in the direction of the function we are learning from (cf. GBM05, LK06).

\section{Concluding Remarks}

We have compared the opening books of humans and computers using nonparametric measures. It seems that there is a strong association between the two books, as the M-measure is over 0.75 and the JSD just above 0.70 , on average. The degree of overlap of move choices 


\begin{tabular}{|c|c|c|c|c|}
\hline Pos & M-measure & MaxM & JSD & Overlap \\
\hline \hline 1 & 0.376 & 3.085 & 0.729 & 0.714 \\
2 & 0.728 & 3.747 & 0.549 & 0.700 \\
3 & 0.634 & 3.597 & 0.633 & 0.778 \\
4 & 0.745 & 3.091 & 0.508 & 0.500 \\
5 & 0.939 & 3.747 & 0.653 & 0.545 \\
6 & 0.534 & 3.940 & 0.486 & 0.462 \\
7 & 0.915 & 3.019 & 0.766 & 0.571 \\
8 & 0.907 & 3.808 & 0.707 & 0.800 \\
9 & 0.672 & 3.293 & 0.765 & 0.444 \\
10 & 0.693 & 3.112 & 0.871 & 0.556 \\
11 & 0.544 & 3.358 & 0.606 & 0.556 \\
12 & 0.896 & 3.747 & 0.637 & 0.545 \\
13 & 0.827 & 2.700 & 0.880 & 0.500 \\
14 & 0.809 & 3.658 & 0.692 & 0.583 \\
15 & 0.376 & 3.658 & 0.479 & 0.462 \\
16 & 0.811 & 3.635 & 0.765 & 0.700 \\
17 & 0.885 & 3.597 & 0.825 & 0.778 \\
18 & 0.955 & 1.850 & 0.716 & 0.500 \\
19 & 0.600 & 3.635 & 0.508 & 0.700 \\
20 & 0.965 & 3.436 & 0.748 & 0.556 \\
21 & 0.978 & 3.019 & 0.863 & 0.833 \\
22 & 0.793 & 3.849 & 0.685 & 0.583 \\
23 & 0.892 & 4.518 & 0.748 & 0.688 \\
24 & 0.846 & 1.083 & 1.000 & 0.333 \\
25 & 0.679 & 3.648 & 0.503 & 0.333 \\
26 & 0.960 & 5.291 & 0.938 & 1.000 \\
\hline Avg & 0.768 & 3.428 & 0.702 & 0.605 \\
Std & 0.175 & 0.776 & 0.144 & 0.157 \\
\hline & & & & \\
\hline
\end{tabular}

Table 5: Summary of results

is just above 0.60 , on average, so despite the correspondence there are also significant differences. Moreover, for the positions we investigated, the expected scores from white's point of view were, on average over $55 \%$, for both humans and machines, which indicates a significant advantage to white for the positions we considered.

More experiments need to be carried out on different test sets covering either a wider range of opening variations or, alternatively, specialising within a small number of popular opening variations. As mentioned in Section f the method we have presented can be used to compare two individuals' opening choices, be they human or machine. Apart from a better understanding of the difference between human and machine players such a comparison may help detect anomalies in an opening book that could be exploited during a match. Finally, the M-measure, or a refinement of it, does not rely on statistics being readily available, so could be used as a similarity measure in learning the evaluation function of an opponent. 


\begin{tabular}{|c|c|r|c|r|}
\hline Pos & Ew\%(pb) & \# pb & Ew\%(comp) & \# comp \\
\hline \hline $1 \mathrm{~b}$ & 51.701 & 298 & 60.217 & 138 \\
$2 \mathrm{w}$ & 54.473 & 474 & 60.418 & 98 \\
$3 \mathrm{w}$ & 52.425 & 414 & 48.529 & 70 \\
$4 \mathrm{w}$ & 53.649 & 285 & 62.944 & 674 \\
$5 \mathrm{w}$ & 57.968 & 1034 & 58.161 & 1810 \\
$6 \mathrm{w}$ & 47.928 & 817 & 52.000 & 111 \\
$7 \mathrm{w}$ & 44.000 & 492 & 47.028 & 2810 \\
$8 \mathrm{~b}$ & 57.616 & 1428 & 59.027 & 297 \\
$9 \mathrm{w}$ & 56.468 & 355 & 64.587 & 1002 \\
$10 \mathrm{w}$ & 56.361 & 2110 & 56.763 & 1606 \\
$11 \mathrm{w}$ & 61.011 & 457 & 68.250 & 72 \\
$12 \mathrm{~b}$ & 57.578 & 296 & 59.258 & 681 \\
$13 \mathrm{w}$ & 50.082 & 743 & 43.656 & 515 \\
$14 \mathrm{w}$ & 62.514 & 849 & 60.501 & 439 \\
$15 \mathrm{w}$ & 57.443 & 140 & 50.573 & 1011 \\
$16 \mathrm{w}$ & 51.387 & 287 & 56.134 & 409 \\
$17 \mathrm{~b}$ & 54.645 & 307 & 53.581 & 501 \\
$18 \mathrm{~b}$ & 59.000 & 76 & 60.277 & 303 \\
$19 \mathrm{w}$ & 55.243 & 202 & 50.396 & 1272 \\
$20 \mathrm{~b}$ & 54.180 & 189 & 53.910 & 297 \\
$21 \mathrm{~b}$ & 55.262 & 420 & 53.740 & 2333 \\
$22 \mathrm{w}$ & 50.613 & 390 & 51.357 & 143 \\
$23 \mathrm{w}$ & 55.029 & 796 & 50.892 & 567 \\
$24 \mathrm{w}$ & 60.000 & 63 & 54.000 & 67 \\
$25 \mathrm{w}$ & 62.005 & 401 & 60.285 & 397 \\
$26 \mathrm{w}$ & 55.036 & 1011597 & 54.749 & 277288 \\
\hline Avg & 55.139 & - & 55.817 & - \\
$\mathrm{Std}$ & 4.301 & - & 5.743 & - \\
\hline
\end{tabular}

Table 6: Expected percentage score

\section{References}

[BML06] J. Bar-Ilan, M. Mat-Hassan, and M. Levene. Methods for comparing rankings of search engine results. Computer Networks, 50:1448-1463, 2006.

[Bur99] M. Buro. Towards opening book learning. International Computer Chess Association Journal, 22:98-102, June 1999.

[Cam99] M. Campbell. Knowledge discovery in Deep Blue. Communications of the ACM, 22:65-67, November 1999.

[CHH02] M. Campbell, A.J. Hoane Jr., and F.-h. Hsu. Deep Blue. Artificial Intelligence, 134:57-83, January 2002. 
[DH97] A.C. Davison and D.V. Hinkley. Bootstrap Methods and their Application. Cambridge Series in Statistical and Probabilistic Mathematics. Cambridge University Press, Cambridge, U.K., 1997.

[DL05a] C. Donninger and U. Lorenz. The Hydra project. Xcell Journal, Issue 53:94-97, Second Quarter 2005.

[DL05b] C. Donninger and U. Lorenz. Innovative opening-book handling. In Proceedings of Conference on Advances in Computer Games, Taipei, Taiwan, September 2005.

[ES03] D.M. Endres and J.E. Schindelin. A new metric for probability distributions. IEEE Transactions on Information Theory, 49:1858-1860, July 2003.

[FKS03] R. Fagin, R. Kumar, and D. Sivakumar. Comparing top $k$ lists. SIAM Journal on Discrete Mathematics, 17:134-160, 2003.

[Für01] J. Fürnkranz. Machine learning in games: A survey. In J. Fürnkranz and M. Kubat, editors, Machines that Learn to Play Games, pages 11-59. Nova Science Publishers, Huntington, NY, 2001.

$\left[\mathrm{GBC}^{+}\right.$02] I. Grosse, P. Bernaola-Galv́an, P. Carpena, R. Román-Rold́an, J. Oliver, and H.E. Stanley. Analysis of symbolic sequences using the Jensen-Shannon divergence. Physical Review E, 65:041905-1-041905-16, 2002.

[GBM05] D. Gomboc, M. Buro, and T.A. Marsland. Tuning evaluation functions by maximizing concordance. Theoretical Computer Science, 2005. To appear.

[GC03] J.D. Gibbons and S. Chakraborti. Nonparametric Statisitcal Inference. Marcel Dekker, New York, NY, fourth edition, 2003.

[Hya99] R.M. Hyatt. Book learning - A methodology to tune an opening book automatically. International Computer Chess Association Journal, 22:3-12, March 1999.

[JAE03] B. Jacobs, J. Aagaard, and J. Emms. Chess Software User's Guide. Everyman Chess, London, U.K., 2003.

[LB05] M. Levene and J. Bar-Ilan. Comparing move choices of chess search engines. International Computer Games Association Journal (ICGA), 28:67-76, 2005.

[Lin00] T.R. Lincke. Strategies for the automatic construction of opening books. In Proceedings International Conference on Computers and Games (CG), pages 7486, Hamamatsu, Japan, 2000.

[LK06] S.M. Lucas and G. Kendall. Evolutionary computation and games. IEEE Computational Intelligence Magazine, 1:10-18, 2006.

[New02] M. Newborn. Deep Blue: An Artificial Intelligence Milestone. Springer Verlag, New York, NY, 2002.

[Pra03] R. Prasad. Man vs. machine - The endless fascination. Www.chessbase.com/ newsdetail. asp?newsid=1296, 2003.

[Sha50] C.E. Shannon. Programming a computer for playing Chess. Philosophical Magazine, 41:256-275, 1950. 